\title{
Temperature distribution and focal depth in the crust of the northeastern Japan
}

\author{
Akiko Tanaka ${ }^{1}$ and Yuzo Ishikawa ${ }^{2}$ \\ ${ }^{1}$ Institute of Geoscience, Geological Survey of Japan, National Institute of Advanced Industrial Science and Technology, Tsukuba 305-8567, Japan \\ ${ }^{2}$ Meteorological Research Institute, Japan Meteorological Agency, Tsukuba 305-0052, Japan
}

(Received December 29, 2001; Revised July 11, 2002; Accepted August 14, 2002)

\begin{abstract}
The thickness of seismogenic crust layer correlates with surface heat flow in most interplate seismic areas of the world (e.g., Sibson, 1982). Although the inverse relationship between heat flow and the base of seismogenic zone is obvious, the quantitative relationships are less certain and there should be variability of the focal depths among different tectonic settings. Comparisons of the heat flow (Yamano et al., 1997), thermal gradient (Tanaka et al., 1999) and earthquake (Japan Meteorological Agency, JMA) databases for the northeastern Japan provide detailed geologic and geophysical information about the earthquake process of island arc. Temperatures in the crust were calculated using a steady-state, one-dimensional, heat conductive transport model with heat generation as a function of heat flow and thermal gradient. The evaluated temperatures for $\mathrm{D}_{90}$, the depth above which $90 \%$ of earthquakes occur, range between $200^{\circ} \mathrm{C}$ and $500^{\circ} \mathrm{C}$ except for high heat flow and thermal gradient data. The consistency of temperature for $\mathrm{D}_{90}$ over a large depth interval supports that the temperature is the dominant factor governing the focal depth in the crust.
\end{abstract}

\section{Introduction}

Temperature has long been regarded as an important variable in determining the seismogenic portion of the lithosphere. Sibson (1982) and others highlighted the general correlation of shallow seismicity with high heat flow and deep seismicity with low heat flow. However, the quantitative relationships are less certain. Heat flow measurements are often widely spaced, requiring an extrapolation of the data to estimate the thermal structure in the crust in some regions. The uncertainties associated with these extrapolations preclude improving on the general correlation between heat flow and depth of seismicity. A new compilation of thermal gradient data around Japan (Yano et al., 1999; Tanaka et al., 1999) yields indirect information about the shallow thermal structure. These new thermal gradient data increase our understanding of the relationship between the thermal regime and the depth limit of seismicity in the crust.

In this paper, we compile, assemble and interpret thermal data beneath the northeastern Japan where both thermal data and seismicity data are relatively well known, in an effort to understand the variations in the thermal regime within the crust. We also estimate the thermal structure of the crust from heat flow and thermal gradient data and compare it with the shallow seismicity.

\section{Distributions of Data}

Ueno et al. (2002) relocated hypocenters of Japan Meteorological Agency (JMA) Earthquake Catalog. We used this re-determined data for the hypocentral distribution. We selected well-determined shallow crustal earthquakes with depth shallower than $20 \mathrm{~km}$ and magnitude greater

Copy right(c) The Society of Geomagnetism and Earth, Planetary and Space Sciences (SGEPSS); The Seismological Society of Japan; The Volcanological Society of Japan; The Geodetic Society of Japan; The Japanese Society for Planetary Sciences. than 1 recorded more than 10 stations between October 1997 and January 2002 and eliminated low-frequency events (Fig. 1(a)). Using this JMA data, the earthquake focal depths are evaluated the depth above which $90 \%$ of earthquakes occur, $\mathrm{D}_{90}$ (e.g., Doser and Kanamori, 1986). We compared this dataset with well-determined focal depths by Tohoku University (Hasegawa and Yamamoto, 1994; Hasegawa et al., 2000). We could not find the difference in $\mathrm{D}_{90}$ between Tohoku University and JMA, even though the observation period is different from that of JMA.

Terrestrial surface heat flow studies and their relationships to other geophysical parameters provide useful insights into the thermal state of the crust. Although the general features of the heat flow distribution in and around Japan had been revealed by the early 1970 s (e.g., Uyeda, 1980), the distribution of heat flow measurements from the recent data compilation (Yamano et al., 1997) is often insufficient to define regional thermal structures (Fig. 1(b)). On the other hand, a new compilation of thermal gradient data around Japan (Yano et al., 1999; Tanaka et al., 1999) yields indirect information about the shallow thermal structure (Fig. 1(c)). This dataset is based on borehole temperature measurements. Thermal gradient was calculated from the difference between the temperature at the bottom of each borehole or the maximum temperature in the borehole and the average surface temperature. Although they have used the borehole data of $300 \mathrm{~m}$ or more in depth, below the transient effects of surface temperature variations and below the groundwater infiltration zone, their thermal gradient data have less accuracy than heat flow data. However, the number and distribution of thermal gradient data is more and wider than heat flow data, and it may be possible to estimate more detailed shallow thermal structure using thermal gradient data.

Figures 2(a) and (b) represent North-South and East-West 

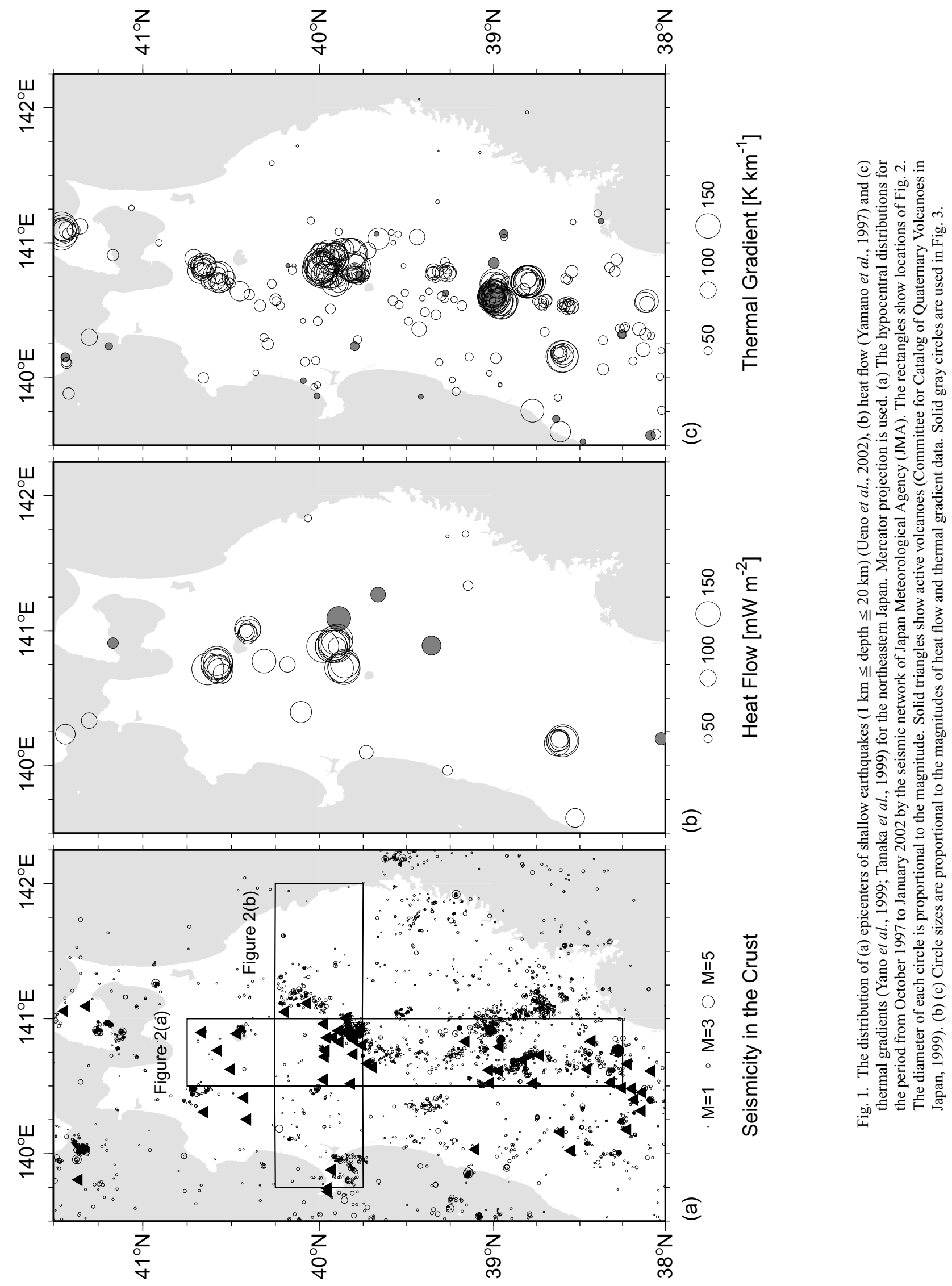

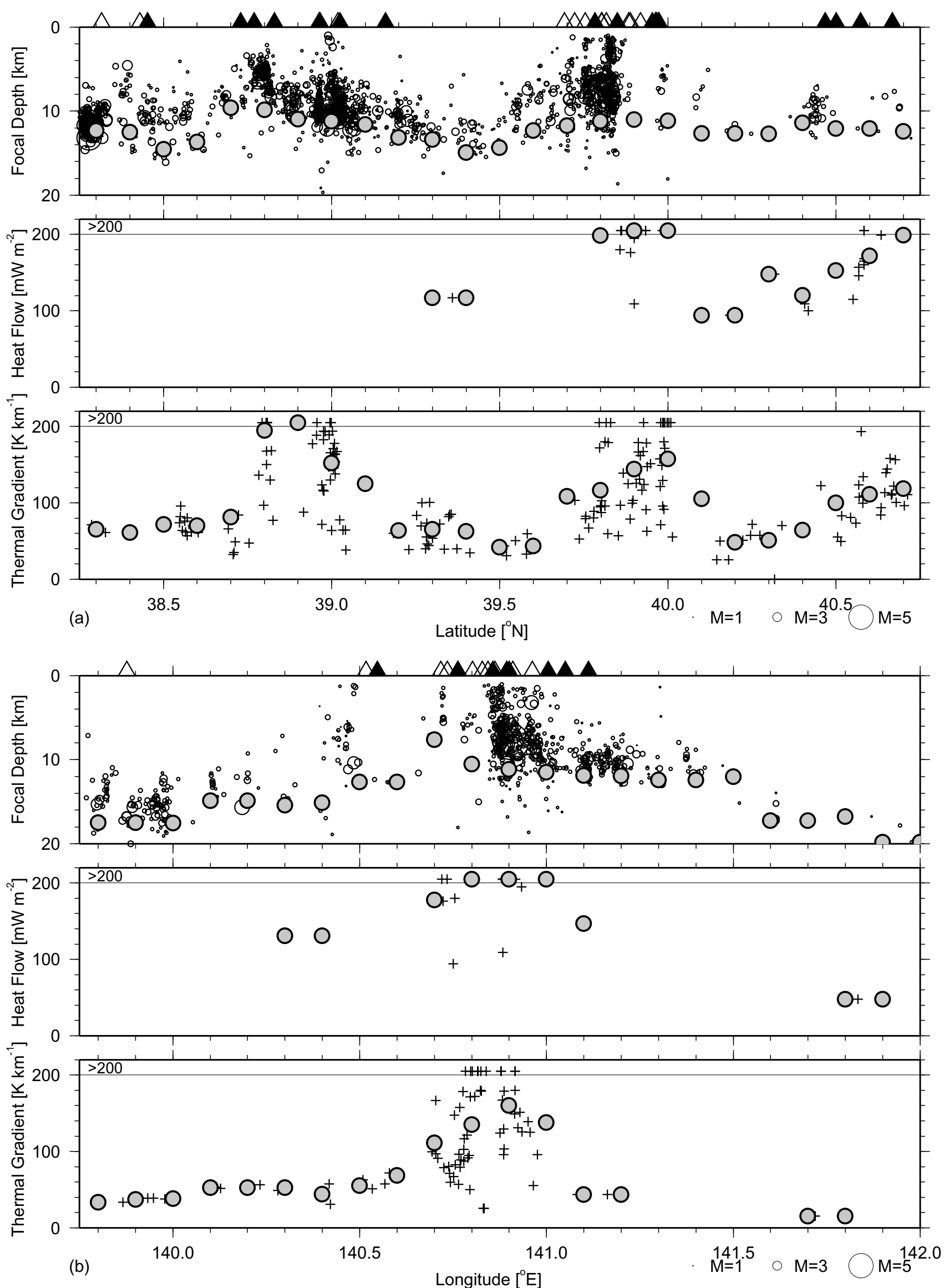

Fig. 2. (a) Cross-section of epicenter (Ueno et al., 2002), heat flow (Yamano et al., 1997), and thermal gradient (Yano et al., 1999; Tanaka et al., 1999) distribution within a distance of $0.25^{\circ}$ across the northeastern Japan along longitude $139.75^{\circ} \mathrm{N}$. Solid and open triangles on the top denote active volcanoes with volcanic products of greater and less than $10 \mathrm{~km}^{3}$, respectively (Committee for Catalog of Quaternary Volcanoes in Japan, 1999). Gray circles show $\mathrm{D}_{90}$ (upper) and moving averages of heat flow (middle) and thermal gradient (lower) every $0.1^{\circ}$. (b) Cross-section along latitude $40^{\circ} \mathrm{E}$. Same conventions as in Fig. 2(a). 

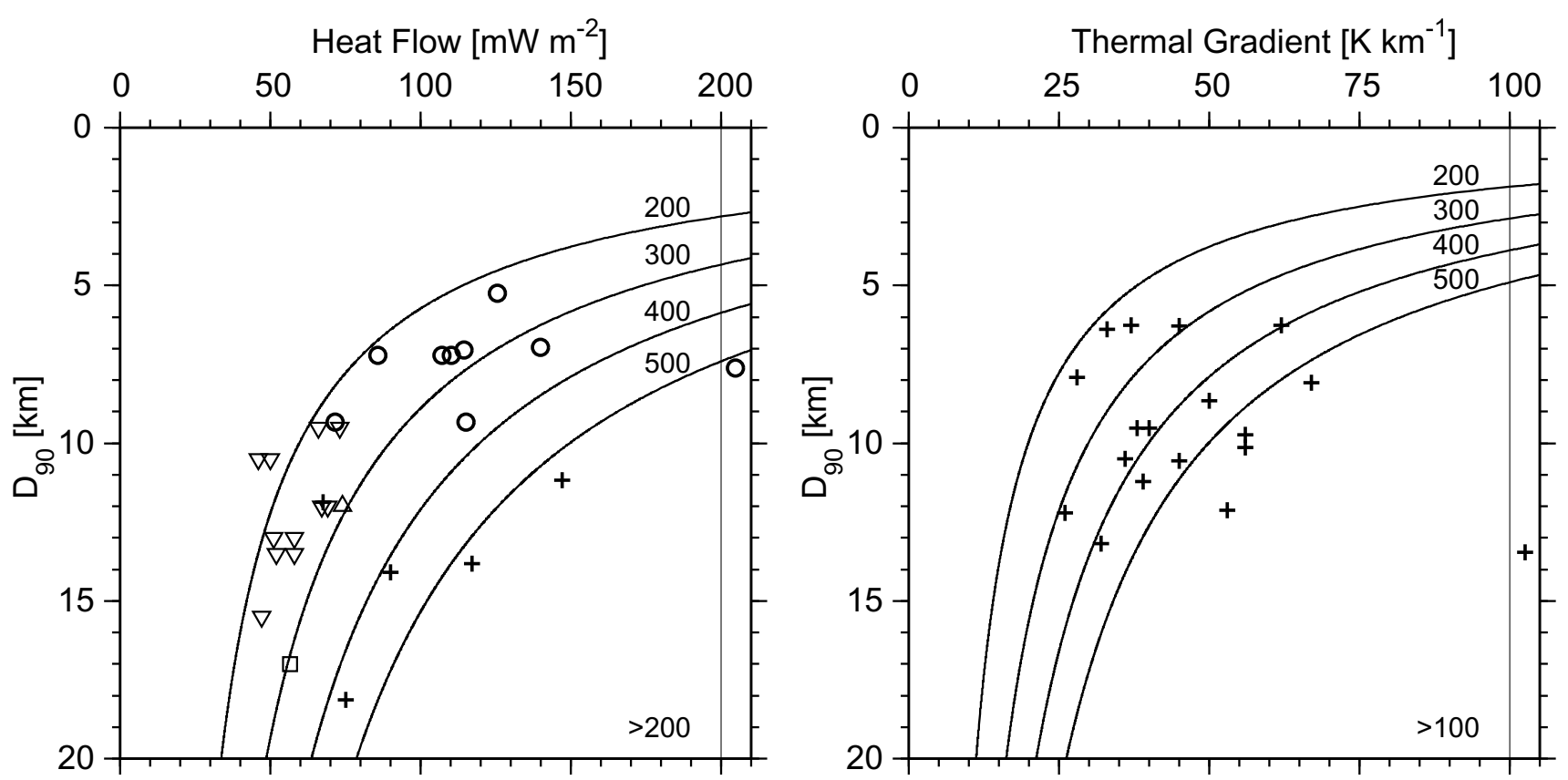

Fig. 3. Plot of $\mathrm{D}_{90}$ against heat flow and thermal gradient data in the northeastern Japan, indicated by crosses. Estimated isotherms of $200^{\circ} \mathrm{C}$ to $500^{\circ} \mathrm{C}$ are shown by solid lines. Previous data since 1990 for heat flow data against maximum focal depth of crustal earthquakes or $\mathrm{D}_{95}$, the depth above which 95\% of earthquakes occur, are also plotted. Seismic and heat flow data are: triangle for KTB (Zoback et al., 1993), inverted triangles for Landers faults (Williams, 1996), square for Nojima fault (Kitajima et al., 2001), circles for the western Nagano Prefecture area (Tanaka and Ito, 2002).

vertical cross-sections in the northeastern Japan, respectively. Figure 2 shows focal depths (upper), heat flow (middle), and thermal gradients (lower) within boxes shown in Fig. 1(a). Gray circles are moving averages of heat flow and thermal gradient data every $0.1^{\circ}$ with $0.2^{\circ}$ intervals. $\mathrm{D}_{90}$ are calculated using focal depths every $0.1^{\circ}$ with $0.2^{\circ}$ intervals shown by gray circles. $\mathrm{D}_{90}$ and averages of heat flow and thermal gradient data are well correlated, although there are some data gaps, especially in heat flow data.

Figure 2(a) shows the cross-section almost along the volcanic front. The depth limit of earthquakes is about $15 \mathrm{~km}$ or less, and it changes remarkably with location. It is locally shallow as $10 \mathrm{~km}$ beneath active volcanoes, indicated by triangles in Fig. 2. Although the resolution of thermal structure by heat flow data is often hindered by a lack of data, heat flow and thermal gradient data well correlate with $\mathrm{D}_{90}$. At almost the same area of Fig. 2(a), Hasegawa et al. (2000) estimated the temperature distribution within the crust from $P$ wave velocity perturbations. Their results are coincident with ours. On the other hand, Fig. 2(b) shows the cross-section almost across the volcanic front. Heat flow and thermal gradients around trench side, the east side, are low, whereas those around the volcanic front are significantly high with large scatter. It is clear that to use thermal gradient data will increase our understanding of the relationship between thermal regime and $\mathrm{D}_{90}$.

\section{Temperatures in the Crust}

In one-dimensional steady-state case under assumptions that the direction of the temperature variation is vertical, Fourier's law takes the form,

$$
k d^{2} T / d z^{2}=-A(z)
$$

where $T$ is the temperature, $k$ is the thermal conductivity, and $A$ is the heat production. Radioactive heat generation cannot be neglected, in calculating the temperatures of a continental-type crust. It has been suggested that radioactive heat generation decreases exponentially with depth in the continental crust (Lachenbruch, 1970). In this case, the temperature is given by

$$
T=T_{0}+\left(\left(q_{0}-A z_{1}\right) / k\right) z+\left(A z_{1}^{2} / k\right)\left(1-\exp \left(-z / z_{1}\right)\right)
$$

$T=T_{0}+\left(\Delta T / \Delta z-A z_{1} / k\right) z+\left(A z_{1}^{2} / k\right)\left(1-\exp \left(-z / z_{1}\right)\right)$

where $q_{0}$ is the heat flux and $\Delta T / \Delta z$ is the thermal gradient. Thermal conductivities, $k$, of continental rocks ranges from $1-5 \mathrm{Wm}^{-1} \mathrm{~K}^{-1}$ (Stein, 1995) and heat production, $A$, of average continental upper crust is $1.0 \mu \mathrm{Wm}^{-3}$ (Fowler, 1990). The characteristic thickness of the layer enriched in radioactive elements has been estimated to be about 10 $\mathrm{km}$. The average temperature of ground surface all over Japan is about $13.51^{\circ} \mathrm{C}$ (National Astronomical Observatory, 1998). We estimate the temperature using the following values, $T_{0}=13.51^{\circ} \mathrm{C}, k=3 \mathrm{Wm}^{-1} \mathrm{~K}^{-1}, A=1.0 \mu \mathrm{Wm}^{-3}$, $z_{1}=10 \mathrm{~km}$. Thermal gradient data does not include information about thermal conductivity and lithology, unfortunately. However, it may be reasonable to use the same values as heat flow data and thermal gradient data, in case of deep reliable borehole data.

\section{Discussions and Conclusion}

Figure 3 shows the correlation of $\mathrm{D}_{90}$ with heat flow and thermal gradient values. The inverse relationship between heat flow or thermal gradient and $\mathrm{D}_{90}$ is obvious. We choose 
heat flow data that has information about thermal conductivity and thermal gradient data whose standing time of longer than 6 hours and depth of deeper than $1000 \mathrm{~m}$. We calculate $\mathrm{D}_{90}$ using seismic events within a distance of $0.05^{\circ}$. We estimate the temperatures using heat flow data (Eq. (11)), and thermal gradient data (Eq. (1-2)). The estimated temperatures are shown as isotherms in Fig. 3. Variations in the evaluated temperatures for $\mathrm{D}_{90}$ range between $300^{\circ} \mathrm{C}$ and $500^{\circ} \mathrm{C}$ in case of heat flow data. We also plot the previous studies in various regions in Fig. 3(a). Compilation of our results and previous studies show that the evaluated temperatures for $\mathrm{D}_{90}$ range between $200^{\circ} \mathrm{C}$ and $500^{\circ} \mathrm{C}$ with some exceptions, although isotherms change depending on assumed equations and parameters. It is shown that the temperatures are higher in the northeastern Japan. It may result in the geological context. We used heat flow data in the areas where Late Oligocene volcanic and sedimentary rocks cover pre-Tertiary basement rocks and are overlain by Quaternary volcanic rocks (Editorial Committee for the Geology of the Japanese Islands, 2002).

In case of thermal gradient data (Fig. 3(b)), most of the evaluated temperatures for $\mathrm{D}_{90}$ range between $200^{\circ} \mathrm{C}$ and $550^{\circ} \mathrm{C}$ except for some exceptions. Figure 3(b) shows considerable scatter, although $\mathrm{D}_{90}$ diminish with decreasing thermal gradient data. Our data shows higher temperature, and this may be explained in lithology of this area. Very high thermal gradient values may not reflect the thermal structure in the crust and may reflect the thermally significant fluid flow and volcanic and geothermal activities. Another reason for this discrepancy comes from the estimation of $\mathrm{D}_{90}$. We used the seismic events within a distance of $0.05^{\circ}$ and this distance cannot reflect a locally shallow focal depth. In some areas, sparse seismicity of the target area offers little information.

Thermal data with seismicity catalogues of re-determined and well-located events can be used to investigate the thermal influence on the depth extent of seismicity in the crust. We compile a data set of $\mathrm{D}_{90}$ versus corresponding heat flow and thermal gradient data. Although it is difficult to estimate the absolute values of the $\mathrm{D}_{90}$ temperature, the consistency of temperature for $\mathrm{D}_{90}$ over a large depth interval supports that the temperature is the dominant factor governing the focal depth in the crust. It also suggests that the pattern of the heat flow and thermal gradient data is useful as an index of the thermal structure in the crust and $\mathrm{D}_{90}$. A comparison of our results with other tectonic regions could provide evidence for variations in temperatures for $\mathrm{D}_{90}$.

Acknowledgments. We wish to thank Colin F. Williams and Kiyoshi Ito for their helpful comments and constructive reviews. We thank members of related organization (Ministry of Education, Culture, Sports, Science and Technology, National Research Institute for Earth Science and Disaster Prevention, Hokkaido University, Hirosaki University, Tohoku University, University of Tokyo, Nagoya University, Kyoto University, Kochi University, Kyusyu University, Kagoshima University, National Institute of Advanced Industrial Science and Technology, Tokyo Prefecture, Hot Springs Research Institute of Kanagawa Prefecture, Shizuoka Prefecture, Yokohama City, JAMSTEC, and JMA) of which high sensitivity seismogram data are transmitted to JMA, who operate the routine observation network, for using valuable data in this study. We also thank A. Hasegawa and N. Umino of Tohoku University for making their well-determined hypocenters available. All figures were prepared with Generic Mapping Tools developed by Wessel and Smith (1995). This study is a part of the Comprehensive ressearch program on flow and slip processes in and below the seismogenic region sponsored by Ministry of Education, Culture, Sports, Science and Technology (MEXT).

\section{References}

Committee for Catalog of Quaternary Volcanoes in Japan (ed.), Catalog of Quaternary volcanoes in Japan, The Volcanological Society of Japan, 1999.

Doser, D. I. and H. Kanamori, Depth of seismicity in the Imperial Valley region (1977-1983) and its relationship to heat flow, crustal structure, and the October 15, 1979, earthquake, J. Geophys. Res., 91, 675-688, 1986.

Editorial Committee for the Geology of the Japanese Islands (ed.), Computer Graphics: Geology of the Japanese Islands (CD-ROM Version), 2002.

Fowler, C. M. R., The Solid Earth, 472 pp., Cambridge University Press, 1990.

Hasegawa, A. and A. Yamamoto, Deep, low-frequency microearthquakes in or around seismic low-velocity zones beneath active volcanoes in the northeastern Japan, Tectonophys., 233, 233-252, 1994.

Hasegawa, A., A. Yamamoto, N. Umino, S. Miura, S. Horiuchi, D. Zhao, and $\mathrm{H}$. Sato, Seismic activity and deformation process of the overriding plate in the northeastern Japan subduction zone, Tectonophys., 319, 225239,2000

Kitajima, T., Y. Kobayashi, R. Ikeda, Y. Iio, and K. Omura, Terrestrial heat flow at Hirabayashi on Awaji Island, south-west Japan, The Island Arc, 10, 318-325, 2001.

Lachenbruch, A. H., Crustal temperature and heat production: Implications of the linear heat-flow relation, J. Geophys. Res., 75, 3291-3300, 1970.

National Astronomical Observatory (ed.), Rika Nenpyo (Chronological Scientific Tables), 1058 pp., 1998 (in Japanese).

Sibson, R., Fault zone models, heat flow, and the depth distribution of earthquakes in the continental crust of the United States, Bull. Seism. Soc. Am., 72, 451-463, 1982.

Stein, C. A., Heat flow of the Earth, in Global Earth Physics, A Handbook of Physical Constants, edited by T. J. Ahrens, pp. 144-158, American Geophysical Union, 1995.

Tanaka, A. and H. Ito, Temperature at the base of the seismogenic zone and its relationship to the focal depth of the western Nagano Prefecture area, J. Seism. Soc. Japan, 55, 1-10, 2002 (in Japanese with English abstract). Tanaka, A., Y. Yano, M. Sasada, Y. Okubo, K. Umeda, N. Nakatsuka, and F. Akita, Compilation of thermal gradient data in Japan on the basis of the temperatures in boreholes, Bull. Geol. Surv. Japan, 50, 457-487, 1999 (in Japanese with English abstract).

Ueno, S., S. Hatakeyama, T. Aketagawa, J. Funasaki, and N. Hamada, Improvement of hypocenter determination method by Japan Meteorological Agency, Kenshinjiho, 65, 123-134, 2002 (in Japanese with English abstract).

Uyeda, S., Review of heat flow studies in the Eastern Asia and Western Pacific region. UESCAP, CCOP/SOPAC Tech. Bull., 3, 153-169, 1980.

Wessel, P. and W. H. F. Smith, New version of the Generic Mapping Tools released, EOS Trans. $A G U, \mathbf{7 6}(33), 329,1995$.

Williams, C., Temperature and the seismic/aseismic transition: Observations from the 1992 Landers earthquake, Geophys. Res. Lett., 23, 20292032, 1996.

Yamano, M., M. Kinoshita, and T. Yamagata, Heat flow distribution around the Japanese islands, Chishitsu News, 517, 12-19, 1997 (in Japanese).

Yano, Y., A. Tanaka, M. Takahashi, Y. Okubo, M. Sasada, K. Umeda, and N. Nakatsuka, Geothermal gradient map of Japan, Geological Survey of Japan, 1999 (in Japanese with English abstract).

Zoback, M. D., R. Apel, J. Baumgartner, M. Brudy, R. Emmermann, B. Engeser, K. Fuchs, W. Kesseks, H. Rischmuller, F. Rummel, and L. Vernik, Upper crustal strength inferred from stress measurements to 6 $\mathrm{km}$ depth in the KTB borehole, Nature, 365, 633-635, 1993.

A. Tanaka (e-mail: akiko-tanaka@aist.go.jp) and Y. Ishikawa 